\title{
The Realization of Fuzzy PID Algorithm in Air Conditioning Control System
}

\author{
Zhao Wei, Li Fangyun, Ge Jing \\ Institute of Technology, East China Jiaotong University, Nanchang Jiangxi, 330100, China
}

Keywords: air conditioning; control system; fuzzy algorithm; PID controller

\begin{abstract}
The application of fuzzy PID algorithm in air conditioning control system not only offers the latter a chance to adjust parameters intelligently, but also helps to get the capability of intelligent frequency modulation. This paper hereby explains the realization methods of fuzzy PID algorithm in air conditioning control system.
\end{abstract}

The air conditioner uses the principle of cold and heat exchange to achieve the effect of space cooling while the adjustment of the parameters and the completion of the refrigeration control process is the air conditioning control system.

\section{The Application Target of Fuzzy PID Algorithm in Air Conditioning Control System}

When using the air conditioning, if the frequency conversion speed regulation of the air conditioner can be intelligently performed and the parameters can be intelligently controlled, then the cooling effect will be optimized and the energy will be saved. However, there are some problems in the design of the current intelligent variable frequency speed control air conditioning system. First, it is difficult to establish an intelligent inverter air-conditioner model as the using of air conditioner will be affected by various factors. For example, external environment and the number of people in using space both influence the cooling effect. But it is difficult for general mathematical calculation model to analyze and deal with a large number of variable factors. Second, the mathematical model in air conditioning control system is a kind of nonlinear model, which brings difficulties to the control processing. Although some nonlinear models can be transformed into linear models, there will be deviations in the calculated results in those complex nonlinear models after simplified, so as to deviates the control process. Then in order to optimize the air conditioning control system, the fuzzy PID algorithm can be applied to improve its control effect.

\section{Fuzzy Algorithm Theory}

\subsection{The fuzzy set theory}

When fuzzy concept is not included, the deterministic eigenfunctions will be applied to analyze the elements in the set. For example, when discussing the characteristic function of set A, $f_{A}(x)=\left\{\begin{array}{ll}1, & x \in A \\ 0, & x \notin A\end{array}(1)\right.$; Equation (1) is used to explore it. It discusses whether a variable $x$ belongs to the element in set $\mathrm{A}$ or not and it can be determined that the variable $x$ can only be inside the set A or outside the set A. However, there is a problem in real life, that is, a variable $x$ may belong to the set A, or may not belong to the set $\mathrm{A}$, but the function attribute of the variable cannot be determined. At this time, if the fuzzy concept is applied to explore the attribute of the variable, only the degree of the variable can be judged. Then, the concept of the fuzzy set is applied to describe the function characteristic attribute of the variable, and Equation (2) $f_{A}(x)=\left\{\begin{array}{l}1, x \in A \\ (0,1) x \in A \text { (2) is } \\ 0, x \notin A\end{array}\right.$ used here. In Equation (2), the closer the data of the variable $x$ is more close to $0, x$ deviates more to set $\mathrm{A}$, and the closer to 1 means the more biased to belong to the set A. Fuzzy set theory is an 
extension of the set theory. The set theory also uses intersection, union and complement methods to explore the logic of elements in a set, but its calculation have multi-valued logic, and the attributes of variables in the set have the possibility of superposition.

\subsection{Calculation method of fuzzy set}

\subsubsection{Binary fuzzy representation of fuzzy set}

If there are two fuzzy sets, the binary fuzzy representation method is described in Equation (3). In Equation (3), $R$ is the universe of discourse of the fuzzy set, that is, the scope of the fuzzy set; in fuzzy set $\mathrm{A}$, there is one variable $x$, and in fuzzy set $\mathrm{B}$, there is a variable $y$. The binary fuzzy set explores the result of the interaction between the variable $x$ in fuzzy set A and the variable $y$ in fuzzy set B. This result will be a fuzzy result.

$$
R=\int_{A \times B} \mu_{R}(x, y) /(x, y) \quad x \in A, y \in B
$$

\subsubsection{Establishment of binary fuzzy set matrix}

In set theory, the matrix of the binary relationship will be applied to calculate the result of the interaction of two variables in two sets, which can also be applied to the establishment of the binary relationship of fuzzy set, but the data result calculated by the binary matrix is also the value of a value of fuzzy program. The fuzzy matrix is now used to analyze the binary relation in Equation (3). The existing set $A=\left\{x_{1}, x_{2}, \cdots, x_{n}\right\}_{\text {and }} B=\left\{y_{1}, y_{2}, \cdots, y_{m}\right\}$ is a finite set, and the fuzzy relation $R$ is applied to describe the mapping result $n \times m$ in the binary relation $A \times B$. Equation (4) can be obtained.

$$
R(A, B)=\left[\begin{array}{cccc}
\mu_{R}\left(x_{1}, y_{1}\right) & \mu_{R}\left(x_{1}, y_{2}\right) & \cdots & \mu_{R}\left(x_{1}, y_{m}\right) \\
\mu_{R}\left(x_{2}, y_{1}\right) & \mu_{R}\left(x_{2}, y_{2}\right) & \cdots & \mu_{R}\left(x_{1}, y_{m}\right) \\
\vdots & \vdots & & \vdots \\
\mu_{R}\left(x_{n}, y_{1}\right) & \mu_{R}\left(x_{n}, y_{1}\right) & \cdots & \mu_{R}\left(x_{n}, y_{m}\right)
\end{array}\right]
$$

The value in Equation (4) is a fuzzy value whose range is $[0,1]$.

\subsubsection{Methods of binary fuzzy set operation}

Now assume there is a direct product space with two fuzzy sets in it, which are described as $X \times Y=\{(x, y) \mid x \in X, y \in Y\}$. The relationship between the two fuzzy sets is described as a fuzzy subset $R$, which $R(x, y)$ can be applied to describe the degree of relationship between the fuzzy subset $R$ and the elements $x$ in the set $X$ and the elements $y$ in the set $Y$ in the direct product space, which applies the arithmetic logic of intersection, union, and complement in the set theory.

When describing the correspondence between $\mathrm{X} \times \mathrm{Y}$ and $\mathrm{A} \rightarrow \mathrm{B}$ in $R(x, y)$, a set of logical reasoning relationships are needed to describe it. At present, the more common logical relationship are "if-then", "else", "also" and so on. If the logical relationship is different, the calculation method is different too. Taking the logical relationship calculation of "if-then" as an example, there are four different operation methods and while the focus of the four methods are different, the calculation results of these four operation methods are different. When calculating the binary fuzzy set relationship, the most suitable operation formula should be chosen according to the different needs of the discussion.

\subsubsection{Establishment of binary fuzzy set calculation}

After the calculation logic and the operation rules of the fuzzy subset $R$ are determined and the value is calculated, the value of $R(x, y)$ is a range of values ranging from[0,1]. Therefore, a rule should be created for the binary fuzzy set calculation, which will describe the range of values of 
$R(x, y)$. Each of the ranges of values corresponds to a control operation. The calculated value of $R(x, y)$ is in a range, and the control decision corresponding to the range is applied to complete the operation.

\section{The Application of PID Controller}

\subsection{The algorithm principle of PID controller}

PID controller is a kind of component that is often used in smart devices. It is a component with intelligent control function that applies the PID control principle to conduct the deviation adjustment of the entire control system so that the controlled value is as close as possible to the actual value.

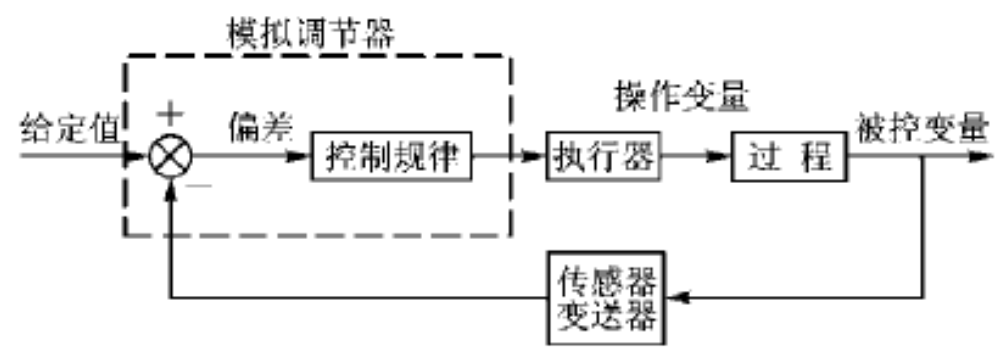

Figure 1: The basic feedback control loop of PID controller

In Figure 1, the PID controller optimizes the control of the air conditioner by controlling the given value and the controlled variable, so that the adjustment of the parameters of the air conditioner can reach the target of the given value. The application of the PID controller enables the air conditioning system to intelligently control parameters to achieve the function of intelligent frequency conversion speed regulation of the air conditioner. The PID of PID controller is an abbreviation of proportion (P), integration (I), and differentiation (D). PID uses a linear combination method to combine these three values to obtain a control variable, which is the numerical basis of the controlled decision of the controlled object.

\subsection{The algorithm equation of PID controller}

In PID controller, the algorithm design of analog regulator is the focus of PID controller function. When PID controller accepts the given value, it can calculate the deviation of the control according to the deviation between the value of the current controlled variable and the given value, and then adjust parameters according to the deviation.

PID controller is divided into proportional controller, proportional integral controller, proportional differential controller, and proportional integral differential controller. The difference of these four controllers is that they only adjust the function of one parameter or adjust the function of multiple parameters. For example, the integral controller only adjusts the role of the integral calculation value, and the proportional integral differential PID controller adjusts the integral, differential, and proportion values. Currently, the most commonly used PID controller is integral differential controller. The algorithm of this controller is now applied to explain how it adjusts parameters. The proportional integral differential PID controller applies Equation (5) to calculate the deviation value and corrects parameters.

The ideal differential equation of the proportional integral differential PID controller is

$$
y=K_{p}\left[e(t)+\frac{1}{T 1} \int e(t) d t+T_{D} \frac{d e(t)}{d t}\right]
$$

In Equation (5), $y$ is the value of controlled variable. The value of controlled variable at a certain time is set as $\mathrm{y}(\mathrm{t})$, the target value required at that time is $\mathrm{r}(\mathrm{t})$, and the deviation between $\mathrm{r}(\mathrm{t})$ and $\mathrm{y}(\mathrm{t})$ is $\mathrm{e}(\mathrm{t})$, and $\mathrm{e}(\mathrm{t})$ is the deviation to be corrected by PID controller. The relation equation of $\mathrm{r}(\mathrm{t}), \mathrm{y}(\mathrm{t})$, and $\mathrm{e}(\mathrm{t})$ is $\mathrm{r}(\mathrm{t})-\mathrm{y}(\mathrm{t})=\mathrm{e}(\mathrm{t})$ ( Equation (6)). Proportional integral differential PID controller achieves the correction of $\mathrm{e}(\mathrm{t})$ by adjusting proportion, integration and differentiation, and outputs $\mathrm{u}(\mathrm{t})$, which is 
the target parameter for the air conditioning to control. The air conditioning control system controls the controlled object, with $\mathrm{u}(\mathrm{t})$ as the target, and finally lets the controlled object output the value $y(t)$. The purpose of applying the proportional integral differential PID controller is to turn the nonlinear model affected by multiple factors into a linear model. The air conditioning system can intelligently complete control decisions by using this kind of model. The control model of the proportional integral differential PID controller based on this algorithm is shown in Figure 2.

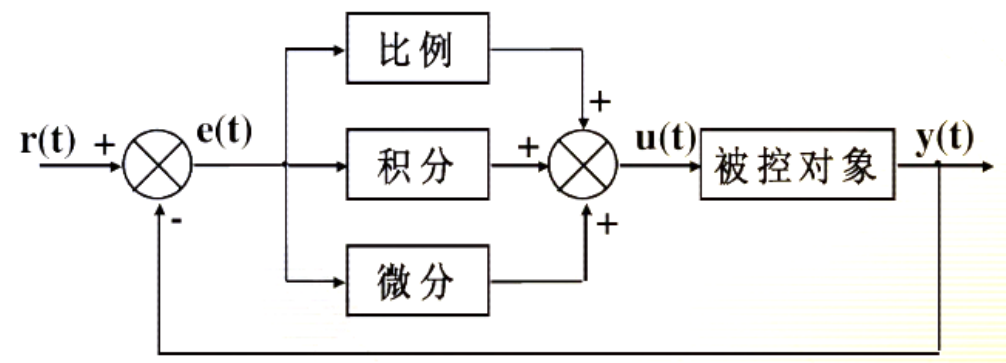

Figure 2: Control model of the proportional integral differential PID controller

The $\mathrm{u}(\mathrm{t})$ output model of the proportional integral differential PID controller is established according to Equation (5) and (6), shown in Equation (7).

$$
u(t)=K_{p}\left[e(t)+\frac{1}{T_{1}} \int_{0}^{t} e(t) d t+T_{D} \frac{d e(t)}{d t}\right]+u_{0}
$$

In Equation (5) (7), e(t) is the value that the controller needs to correct; $\mathrm{u}(\mathrm{t})$ is the output signal given by the regulator; $k_{p}$ is the proportional coefficient; $T_{t}$ is the integral time, $T_{D}$ is the differential time, $U_{0}$ is the control constant, $k_{p} / T_{t}$ is the integral coefficient and $k_{p} / T_{D}$ is the differential coefficient.

\subsection{The regulation function of PID controller}

The purpose of the proportional integral differential PID controller to control the proportional factor is to adjust the proportion of the deviation. The larger the value of $k_{p}$, the larger the adjustment PID controller needs to conduct. However, if $k_{p}$ exceeds a certain range, the hardware facilities of the air conditioning system may not meet the numerical adjustment requirements. If the hardware facilities are forced to operate, the hardware facilities will be damaged. The purpose of PID controller to control the integral coefficient is to correct the accumulated deviation. The larger the value of $k_{p} / T_{t}$, the more accurate the value will be theoretically, and the more stable the air conditioning system will be. However, if the value of $k_{p} / T_{t}$ is too large, the slower the speed of PID controller will be, which will affect the control effect of the air conditioning control system. The purpose of PID controller to control the differential coefficient is to correct the change in deviation. The control of $k_{p} / T_{D}$ can avoid the overclocking of the air conditioning control system during operation and can reduce the large fluctuation in operation. $k_{p} / T_{D}$ is easy to resonate with the noise of input signal, so sometimes the air conditioning control system does not apply the control value of $k_{p} / T_{D}$ to adjust the control, or if the value of $k_{p} / T_{D}$ is used to adjust the control, the filter system is selected to be installed in the air conditioner.

\subsection{Discrete calculation of PID controller}

Theoretically, the correction of the air conditioning control system can be achieved with Equation (7). However, in practical use, discrete data acquisition is the problem faced by PID controller because when using PID controller, the data at the input of PID controller is collected by the computer, and when the computer collects data, there may be a discontinuity. Thus, where the data acquisition is discontinuous, there are problems in the calculation of the integration and the differentiation. So a set of discrete rules must be created to handle discontinuous data. The discrete 
equation for PID controller is shown in Equation (8).

$$
\left.\begin{array}{l}
t \approx k T \quad k=(0,1,2,3, \ldots) \\
\int_{0}^{k} e(t) d t \approx T \sum_{j=0}^{k} e(j T)=T \sum_{j=0}^{k} e_{j} \\
\frac{d e(t)}{d t} \approx \frac{e(k T)-e[(k-1) T]}{T}=\frac{e_{k}-e_{k-1}}{T}
\end{array}\right\}
$$

To facilitate the calculation, Equation (8) is simplified and a discrete PID controller discrete calculation expression is obtained, shown in Equation (9).

$$
u_{k}=K_{P}\left[e_{k}+\frac{T}{T_{1}} \sum_{j=0}^{k} e_{j}+\frac{T_{D}}{T}\left(e_{k}-e_{k-1}\right)\right]+u_{o}
$$

\section{Method to Establish the Air Conditioning Control System with Fuzzy PID Algorithm}

\subsection{The establishment objective}

In order to illustrate the application of fuzzy PID algorithm in the air conditioning control system, an air conditioning control system with constant temperature and humidity is established. Now assume in a space, it is divided into A, B, C, and D area. In the four areas, Area A requires the establishment of a low-temperature and low-humidity environment, Area B requires the establishment of a high-temperature and low-humidity environment, Area $\mathrm{C}$ requires the establishment of a high-temperature and high-humidity environment, and Area D requires the establishment of a low-temperature and high-humidity environment.

\subsection{Implementation method}

\subsubsection{The establishment of fuzzy PID algorithm model}

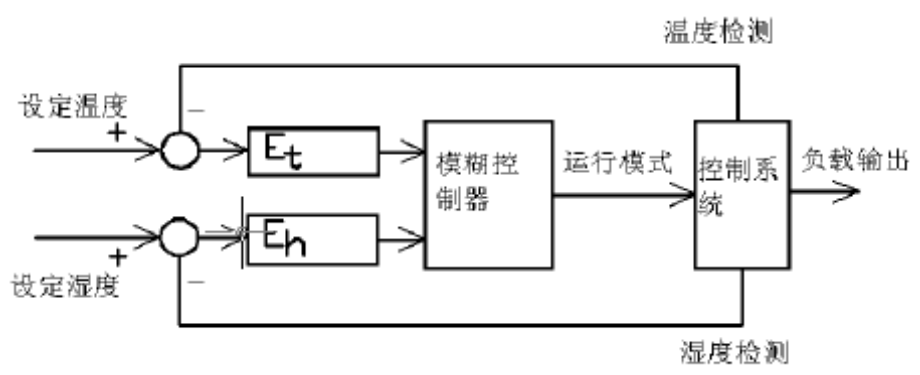

Figure 3 The PID algorithm model of air conditioning control system with constant temperature and humidity

According to the control requirements of the four areas, it can be seen that the parameters needed to be controlled by fuzzy PID algorithm are temperature and humidity, which are adjusted to achieve the purpose of control. Then the fuzzy PID algorithm model is established in Figure 3.

\subsubsection{The fuzzy set principle and the application of fuzzy set algorithm}

It can be seen from the fuzzy PID algorithm model that the temperature values can be described as a set $E_{t}$ and the temperature values as a set $E_{h}$. Then the fuzzy rule is applied to establish the linguistic variables of each set. Set the language variables of the set $E_{h}$ as $\{\mathrm{NCB}, \mathrm{NB}, \mathrm{NM}, \mathrm{NS}, \mathrm{ZE}$, PS, PM, PB, PCB $\}$ and the set field of the set $E_{h}$ is $\{-8,-6,-4,-2,0,2,4,6,8\}$. Set the language variables of the set $E_{h}$ as \{ZE, PS, PM, PB, PCB , and the set field of the set ${ }_{h}$ should be \{-0, 5, $10,15,20\}$. The table of output rule is shown in Figure 4. It can be seen from Figure 4 that the PID algorithm gives four control decision schemes. 


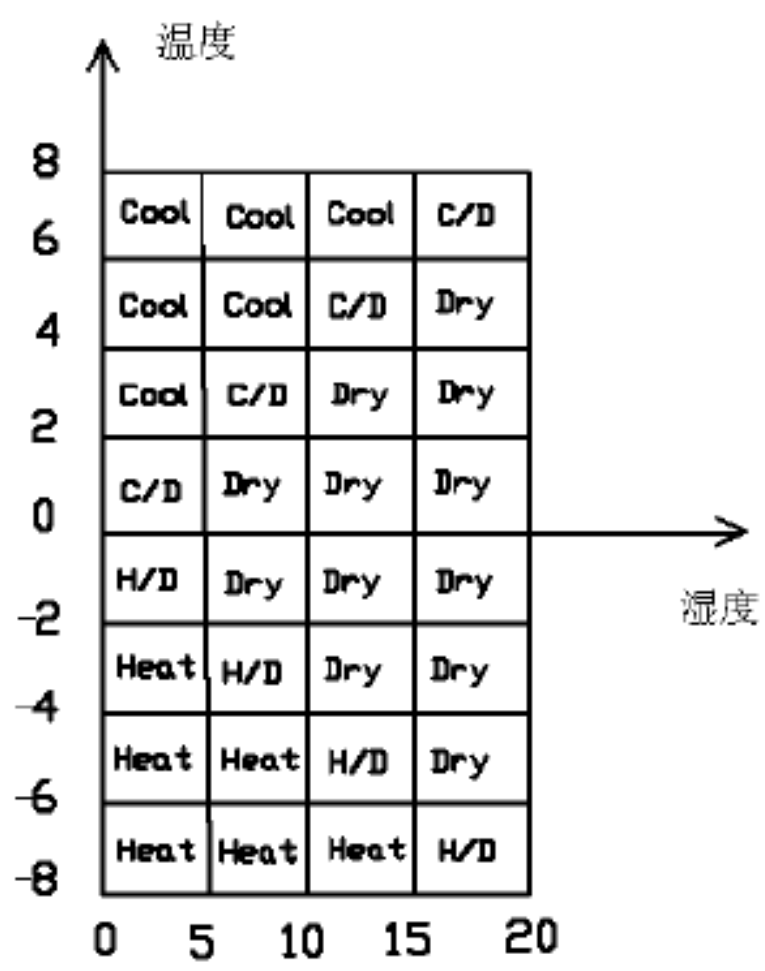

Figure 4: The PID algorithm output rule control table of the air conditioning control system with constant temperature and humidity

\subsubsection{Decoupling algorithm design of the control target}

If there is only a variable of temperature or humidity to be controlled, then no decoupling analysis is required. The control of constant temperature and humidity requires the control of two variables, temperature and humidity. There is a coupling relationship between these two variables. That is, when the temperature variable changes, the humidity variable also changes, so the coupling relationship between the two variables needs to be analyzed. The fuzzy operation rules and coupling structure of temperature and humidity are shown in Figure 5.

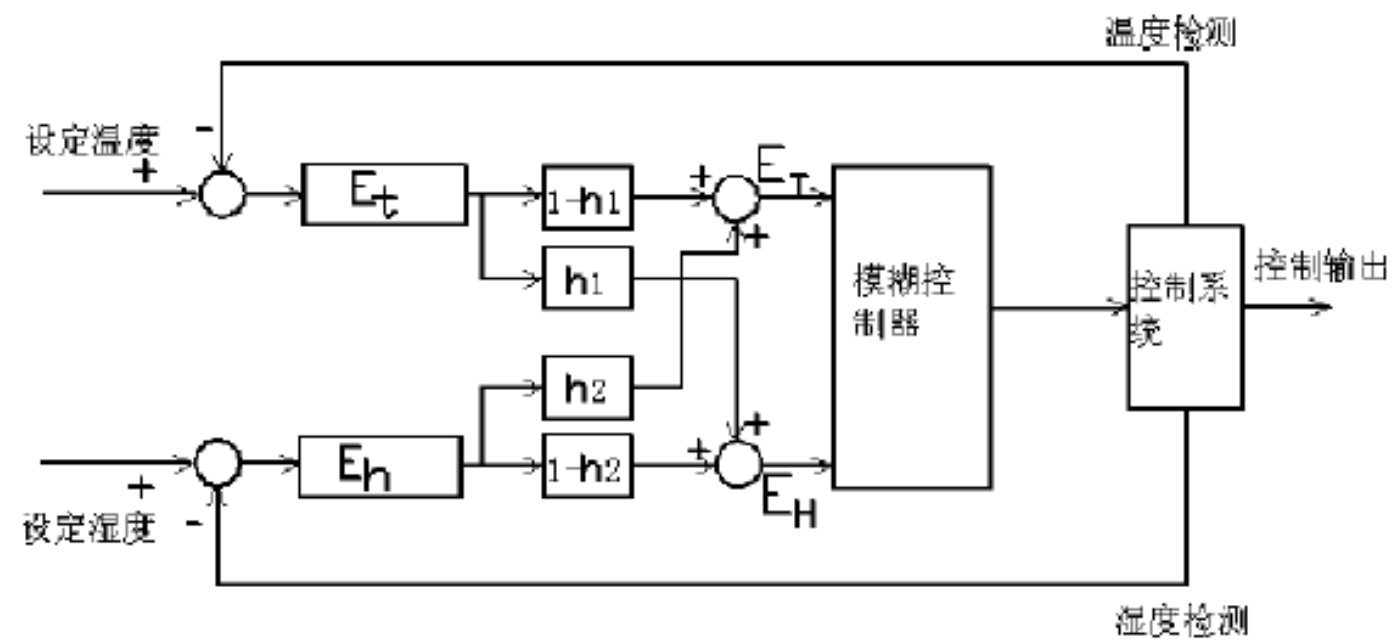

Figure 5: The fuzzy operation rules and coupling structure of air conditioning control system with constant temperature and humidity

The calculation of the temperature is Equation (10): $\mathrm{E}_{\mathrm{T}}=\left[\left(1-h_{1}\right) \times \mathrm{E}_{\mathrm{t}}+h_{1} \times \mathrm{E}_{\mathrm{h}}\right]$ (10);

The calculation of the humidity is Equation (11): $\mathrm{E}_{\mathrm{H}}=\left[\left(1-h_{2}\right) \times \mathrm{E}_{\mathrm{h}}+h_{2} \times \mathrm{E}_{\mathrm{t}}\right]$ (11);

The coupling relationship between Equation (10) and (11) is analyzed. When both $h_{1}$ and $h_{2}$ are 0 , then $E_{T}=E_{t}, E_{H}=E_{h}$; when $0<h_{1}, h_{2}>0$, when both $h_{1}$ and $h_{2}$ are 1 , then $E_{T}=E_{h}, E_{H}=E_{t}$, which is a 
full coupling. In practical use, the coupling relationship between temperature and humidity is neither 0 nor 1 , so only one value that matches the relationship between temperature and humidity can be taken. By applying a coupling coefficient of temperature and humidity, it is possible to set a temperature or humidity target to create one of the four environments A, B, C, or D.

\subsubsection{The establishment of fuzzy PID algorithm calculation equation}

The fuzzy PID algorithm is applied to establish the simulation form and discretization form of the air conditioning control system. The calculation process of the simulation form is $e(t)=r(t)-c(t), \frac{d e(t)}{d T}, \int_{0}^{t} e(t) d t$. The form of discretization is $e(n)=r(n)-c(n)$,
$\frac{e(n)-e(n-1)}{T} \sum_{i=0}^{n} e(i) T=T \sum_{i=0}^{n} e(i)$. These three steps are the principle of the application of Equation (6). The constant temperature wet discretization model is established according to Equation (9).

$$
u(n)=K_{P}\left\{e(n)+\frac{T}{T_{I}} \sum_{i=0}^{n} e(i)+\frac{T_{D}}{T}[e(n)-e(n-1)]\right\}+u_{0}
$$

The proportional term is established according to Equation (12): $u_{P}(n)=K_{P} e(n)$;

The integral term is established according to Equation (12):

$$
u_{I}(n)=K_{P} \frac{T}{T_{I}} \sum_{i=0}^{n} e(i)
$$

The differential term is established according to Equation (12):

$$
u_{D}(n)=K_{P} \frac{T_{D}}{T}[e(n)-e(n-1)]
$$

Now, the value of $u(n)$ can be adjusted through the adjustment of the proportion, the integration, and the differentiation and they are regarded as the target data for the controlled object.

\subsection{The simulation results of the air conditioning control system established by fuzzy PID algorithm}

In order to explain the effectiveness of fuzzy PID algorithm, the simulation experiments are now used to illustrate. Create a simulation space using MatLab's Simulink tool. The error switching point is set as $|e|=5$. When using the air conditioner, two parameters cannot be set at the same time, and only one parameter can be set at a time. The room temperature is now set to $22{ }^{\circ} \mathrm{C}$, the target temperature is $20^{\circ} \mathrm{C}$, the limiter parameter value is 3 , the delay is $20 \mathrm{~s}$, and the simulation time is 600 s. It can be seen from the simulation results in Fig. 6 that after 180 s, the state of constant temperature is reached.

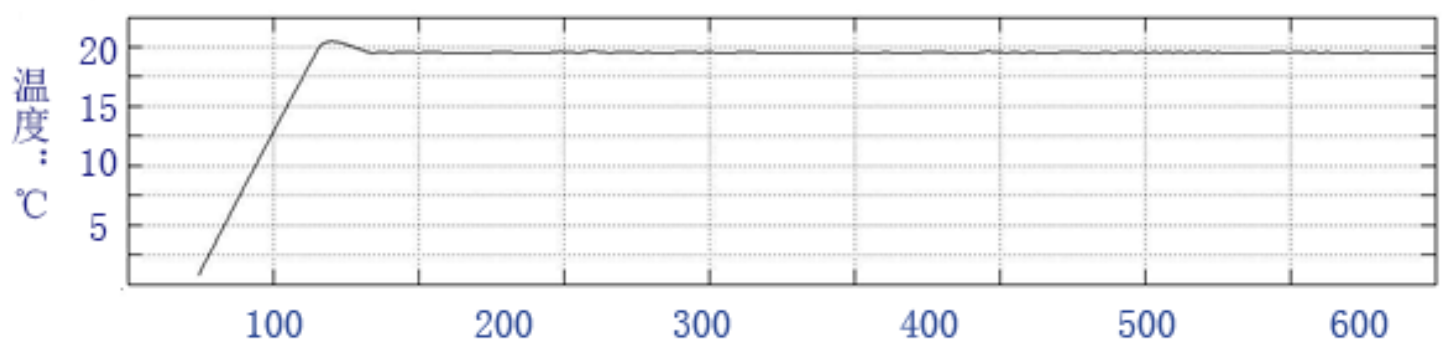

Figure 6: The adjustment effect of air conditioning control system with constant temperature and humidity established by fuzzy PID algorithm (1)

The temperature control effect at the same time is shown in Fig. 7. As can be seen from Fig. 7, after $80 \mathrm{~s}$, the humidity is controlled at $30 \%$. 


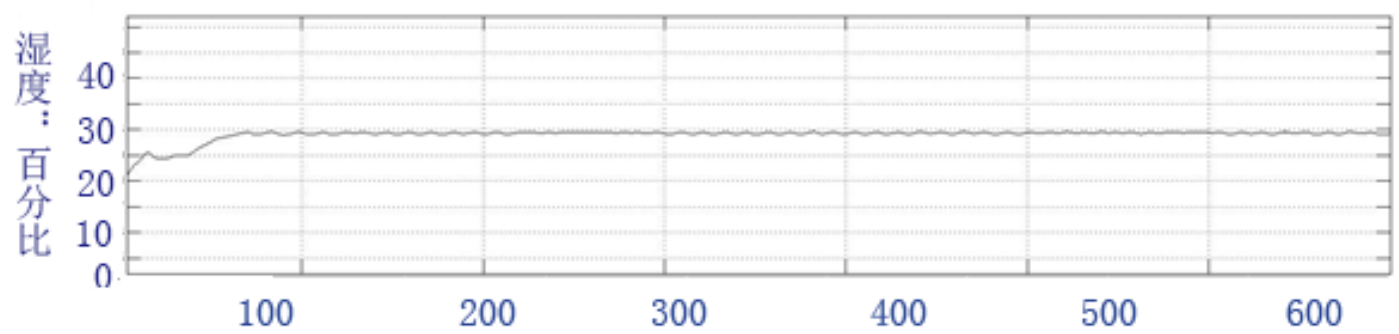

Figure 7: The adjustment effect of air conditioning control system with constant temperature and humidity established by fuzzy PID algorithm (2)

After applying the fuzzy PID algorithm in air conditioning control system with constant temperature and humidity, the air conditioner has the function of intelligent frequency modulation and speed regulation, and there is fast response, small vibration and low volatility.

\section{Conclusion}

The fuzzy PID algorithm is applied in the air conditioning control system. PID controller can be used to simplify the nonlinear influences of multiple factors, and thus the air conditioning control problem becomes linear problem. By applying fuzzy calculation, the relevant parameters can be intelligently adjusted and the control of the target parameters such as the temperature and humidity of the air conditioner can be intelligently completed.

\section{Acknowledgement}

Fund Project: This paper comes from the Science and Technology Research Project of Department of Education in Jiangxi Province. Project Name: Research on Ice Storage Air Conditioning Control System Based on Fuzzy Control. Project No.: 161560

\section{References}

[1] Yang Fan, Chen Maolin, Ma Xinwen, Zhang Hao. Purification Air Conditioning Monitoring System Based on Fuzzy PID Algorithm [J]. Journal of Huazhong University of Science and Technology (Natural Science). 2014(01)

[2] Liu Jingwei, Wei Dong, Dai Zhengwei. Research and Implementation of VAV Control System Based on Fuzzy PID Control [J]. Journal of Beijing Institute of Technology. 2010(08)

[3] Tang Xin, Zuo Weiheng, Li Changchun. Simulation Research on Temperature PID Control of Central Air Conditioning Room [J]. Computer Simulation. 2010(05) 